\title{
Fibroblast growth factor 15/19 (FGF15/19) protects from diet-induced hepatic steatosis: development of an FGF19-based chimeric molecule to promote fatty liver regeneration
}

\author{
Gloria Alvarez-Sola, ${ }^{1}$ Iker Uriarte, ${ }^{1}$ M Ujue Latasa, ${ }^{1}$ Maite G Fernandez-Barrena, ${ }^{1}$ \\ Raquel Urtasun, ${ }^{1}$ Maria Elizalde, ${ }^{1}$ Marina Barcena-Varela, ${ }^{1}$ Maddalen Jiménez, ${ }^{1}$ \\ Haisul C Chang, ${ }^{1}$ Roberto Barbero, ${ }^{1}$ Victoria Catalán, ${ }^{2}$ Amaia Rodríguez, ${ }^{2}$ \\ Gema Frühbeck, ${ }^{2}$ José M Gallego-Escuredo, ${ }^{3}$ Aleix Gavaldà-Navarro, ${ }^{3}$ \\ Francesc Villarroya, ${ }^{3}$ Carlos M Rodriguez-Ortigosa, ${ }^{1}$ Fernando J Corrales, ${ }^{1}$ \\ Jesus Prieto, ${ }^{1}$ Pedro Berraondo, ${ }^{4}$ Carmen Berasain, ${ }^{1}$ Matias A Avila ${ }^{1}$
}

\begin{abstract}
Additional material is published online only. To view please visit the journal online (http://dx.doi.org/10.1136/ gutjnl-2016-312975).
\end{abstract}

${ }^{1}$ Hepatology Programme, CIMAUniversity of Navarra, IdiSNA, CIBEREHD, Pamplona, Spain ${ }^{2}$ Metabolic Research Laboratory, Clínica Universidad de Navarra, IdiSNA, CIBEROBN, Pamplona, Spain

${ }^{3}$ Department of Biochemistry and Molecular Biology, University of Barcelona, CIBEROBN, Barcelona, Spain ${ }^{4}$ Immunology Programme, CIMA-University of Navarra, IdisNA, Pamplona, Spain

Correspondence to Professor Matias A Avila and Carmen Berasain. CIMAUniversity of Navarra, Avda. Pio XII, n55. Pamplona 31008, Spain; maavila@unav.es; cberasain@unav.es

MAA and $C B$ share senior authorship. GA-S and IU made equal contribution to the study.

Received 31 August 2016 Revised 27 December 2016 Accepted 3 January 2017 Published Online First 24 January 2017

\section{SLinked}

- http://dx.doi.org/10.1136/ gutjnl-2017-313712

CrossMark

To cite: Alvarez-Sola G, Uriarte I, Latasa MU, et al. Gut 2017:66:1818-1828.

\section{ABSTRACT}

Objective Fibroblast growth factor 15/19 (FGF15/19), an enterokine that regulates synthesis of hepatic bile acids (BA), has been proposed to influence fat metabolism. Without FGF15/19, mouse liver regeneration after partial hepatectomy $(\mathrm{PH})$ is severely impaired. We studied the role of FGF15/19 in response to a high fat diet (HFD) and its regulation by saturated fatty acids. We developed a fusion molecule encompassing FGF19 and apolipoprotein A-I, termed Fibapo, and evaluated its pharmacological properties in fatty liver regeneration. Design $\mathrm{Fgfl}^{-}{ }^{-}$mice were fed a HFD. Liver fat and the expression of fat metabolism and endoplasmic reticulum (ER) stress-related genes were measured. Influence of palmitic acid (PA) on FGF15/19 expression was determined in mice and in human liver cell lines. In vivo half-life and biological activity of Fibapo and FGF19 were compared. Hepatoprotective and proregenerative activities of Fibapo were evaluated in obese $d b / d b$ mice undergoing $\mathrm{PH}$.

Results Hepatosteatosis and ER stress were exacerbated in HFD-fed Fgf15 ${ }^{-/}$mice. Hepatic expression of Ppar 22 was elevated in $F g f 15^{-/-}$mice, being reversed by FGF19 treatment. PA induced FGF15/ 19 expression in mouse ileum and human liver cells, and FGF19 protected from PA-mediated ER stress and cytotoxicity. Fibapo reduced liver BA and lipid accumulation, inhibited ER stress and showed enhanced half-life. Fibapo provided increased $d b / d b$ mice survival and improved regeneration upon $\mathrm{PH}$.

Conclusions FGF15/19 is essential for hepatic metabolic adaptation to dietary fat being a physiological regulator of Ppar 2 expression. Perioperative administration of Fibapo improves fatty liver regeneration.

\section{INTRODUCTION}

Non-alcoholic fatty liver disease (NAFLD), a spectrum of liver conditions ranging from simple steatosis to steatohepatitis, and eventually progressing to fibrosis and cirrhosis, affects up to $30 \%$ of the general population and $70 \%-90 \%$ of obese

\section{Significance of this study}

What is already known on this subject?

- FGF15/19 is a bile acid (BA)-induced ileumderived enterokine that governs BA homeostasis. Lack of FGF15 results in impaired mouse liver regeneration after partial hepatectomy mainly due to toxic intrahepatic BA concentrations.

- FGF15/19 regulates hepatic glucose metabolism and stimulates protein synthesis.

Pharmacological administration or transgenic expression of FGF19 in mice increases hepatic lipid oxidation, reduces lipogenesis and protects from hepatosteatosis.

- Hepatic steatosis, and the cholestasis associated with this condition, negatively affects experimental and human liver regeneration after hepatectomy or after living donor liver transplantation.

What are the new findings?

- Endogenous FGF15/19 plays a central role in hepatic lipid metabolism. Lack of FGF15 results in increased hepatic steatosis and in the development of endoplasmic reticulum (ER) stress in the liver of mice fed a high fat diet.

- FGF15/19 is a physiological negative regulator of the hepatic expression of the transcription factor Ppary2, which is a major contributor to high-fat diet-induced hepatic steatosis.

- FGF19 gene expression can be activated in parenchymal and biliary human liver cells by ER stress-inducing saturated fatty acids, and FGF19 protects these cells from lipoapoptosis.

- We have developed a new chimaeric molecule encompassing FGF19 and apolipoprotein A-I termed Fibapo with improved pharmacokinetic properties. Fibapo retains FGF19 biological activities on liver BA and fat metabolism and displays potent hepatoprotective and proregenerative effects. 
Significance of this study

\section{How might it impact on clinical practice in the} foreseeable future?

- The FGF15/19-PPAR $\gamma 2$ axis identified in this study may constitute a novel target for the prevention or treatment of hepatic steatosis.

- Engineered FGF19-based molecules such as Fibapo may be applied perioperatively for the improvement of liver regeneration after resection or transplantation, particularly in the presence of hepatosteatosis and cholestasis.

individuals. ${ }^{1}$ The accumulation of hepatic triglycerides (TG) in the cytoplasm of hepatocytes is the histological hallmark of NAFLD. However, TG accumulation per se is not thought to mediate hepatocellular damage, being considered as an adaptive protective response to cope with the increased flux of free fatty acids (FFA) within the liver. ${ }^{1}$ In patients with NAFLD, accumulating hepatic FFAs come from different sources, including the adipose tissue where insulin resistance promotes lipolysis, from de novo lipogenesis and from the diet. ${ }^{1}{ }^{2}$ Peripheral insulin resistance leads to a compensatory hyperinsulinemia, which further drives hepatic de novo lipogenesis. FFAs' excess, and particularly saturated FFAs' accumulation, leads to hepatocellular insulin resistance and lipotoxicity through various mechanisms among which an excessive or prolonged endoplasmic reticulum (ER) stress response is believed to play a key role. ${ }^{1-3}$ Further to ER stress, FFAs may also promote increased synthesis and accumulation of bile acids (BA) leading to hepatocyte injury. ${ }^{4}$ Consistently, elevated hepatic BA levels have been found in patients with NAFLD. ${ }^{5}$ Moreover, FFAs can be also toxic for cholangiocytes, contributing to the development of cholestasis also observed in a subset of patients with NAFLD. ${ }^{6}$

Insulin is indeed essential for the coordination of lipid and carbohydrate metabolism, and hepatic steatosis appears when insulin signalling is impaired in adipose tissue and the liver. ${ }^{1}$ However, recent studies have identified fibroblast growth factor 19 (FGF19; FGF15 in rodents) as an additional nutritionally regulated hormone capable of inhibiting gluconeogenesis and stimulating hepatic glycogen and protein synthesis like insulin, but devoid of the lipogenic effects of this hormone. ${ }^{78}$ FGF15/19 was initially identified as a postprandial endocrine factor released into the portal circulation from ileal enterocytes involved in the inhibition of hepatic BA synthesis. ${ }^{9}$ In the context of diet-induced NAFLD, transgenic expression of FGF19 or its pharmacological administration to obese mice resulted in increased insulin sensitivity and reduced hepatosteatosis. ${ }^{10}{ }^{11}$ Interestingly, a number of reports have found decreased fasting FGF19 serum levels in patients with NAFLD, type 2 diabetes or the metabolic syndrome, as well as an apparent resistance to FGF19 hepatic effects in insulin-resistant patients with NAFLD. ${ }^{12}$ Together, these findings suggest a potential role for FGF15/19 in hepatic lipid homeostasis and highlight its dysregulation in metabolic disorders. However, to our knowledge, the physiological function of this hormone in the response to an obesogenic diet has not been examined. In the first part of this study, we demonstrate the critical role of endogenous FGF15 in the response to diet-induced hepatic steatosis.

While the impact of NAFLD on human health is generally associated with chronic liver disease progression, there are specific circumstances in which the deleterious consequences of NAFLD can manifest acutely. NAFLD negatively affects the outcome of patients undergoing partial liver resection of primary or metastatic liver tumours, or in the setting of liver transplantation. ${ }^{13-15}$ Importantly, the presence of cholestasis, existing prior to resection or developing after liver transplantation, has been associated with steatosis and a worse patient's outcome. ${ }^{13}{ }^{16}$ Experimental studies in the models of NAFLD have also demonstrated the negative impact of this condition on liver regeneration after partial hepatectomy $(\mathrm{PH}) \cdot{ }^{17-20}$ Interestingly, we and others have recently shown that FGF15/19 is important for mouse liver regeneration after $\mathrm{PH}$, contributing to the preservation of BA homeostasis and to parenchymal cells' survival and proliferation. ${ }^{21-23}$ Moreover, we demonstrated that FGF15/19 delivery increased mice survival in a model of extensive hepatectomy, suggesting the therapeutic potential of the perioperative administration of this enterokine. ${ }^{21}$ Therefore, the combined intrinsic biological activities of FGF15/19, namely its ability to lower liver fat, regulate BA levels and promote hepatocellular proliferation, would make this molecule an ideal tool to improve regeneration of steatotic and cholestatic livers. However, FGF15/19 protein has a very short half-life, with a high glomerular filtration rate. ${ }^{24}$ To overcome this limitation, we have developed a new chimaeric molecule based on the fusion of FGF19 with apolipoprotein A-I (ApoA-I) termed Fibapo (FA). As we have previously shown, the ApoA-I moiety confers biological stability and provides liver targeting through its interaction with scavenger receptor class B type I (SR-BI) highly expressed in hepatocytes. ${ }^{25}$ In the second part of this study, we demonstrate the remarkable metabolic and proregenerative activities of FA in a model of fatty liver.

\section{METHODS}

\section{Animals and treatments}

Fgf $15^{-/-}$mice and wild-type littermate controls $\left(F g f 15^{+/+}\right)$have been described before. ${ }^{21}{ }^{26}$ Obese $d b / d b$ mice (C57BL/6 background, male, 8-10 weeks of age) were from Charles River (Barcelona, Spain). $\mathrm{Fgf15} 5^{-/-}$and $\mathrm{Fgfl} \mathrm{5}^{+/+}$mice were fed a high-fat diet (HFD) (60\% of kcal from fat, Research Diets, New Brunswick, New Jersey, USA) for 12 weeks, starting at 8 weeks of age. Body fat content was measured in live conscious animals using quantum molecular resonance (QMR) technology (EchoMRI-100-700, Echo Medical Systems, Houston, Texas, USA). Where indicated, after 14 hours of fasting, mice were subjected to a $500 \mu \mathrm{L}$ bolus p.o of a nutrient-rich diet (Ensure, Abbot Laboratories, $4.6 \mathrm{mg} / \mathrm{g}$ body weight) or of palmitic acid (PA) resuspended in palm oil (2 $\mathrm{mg} / \mathrm{g}$ body weight) (Sigma). Wild-type C57BL/6 mice (8-10 weeks of age) were fasted for 14 hours and then treated with tunicamycin $(100 \mu \mathrm{g} /$ mouse $)$ in a $150 \mathrm{mM}$ sucrose solution p.o. Hydrodynamic injections of plasmids were performed as described. ${ }^{25} \mathrm{PHs}(66 \%$ and $85 \%)$ were performed in mice as described. ${ }^{21}$ Treatment of mice with recombinant FGF19 or FA for the indicated periods of time did not significantly affect weight or food intake. Mice received humane care, and all experiments were carried out in compliance with our institution's ethical guidelines.

Additional Materials and Methods are included as online supplementary files and supplementary tables S1 and S2.

\section{RESULTS}

Lack of FGF15/19 leads to increased adiposity and hepatic steatosis accompanied by ER stress in response to a HFD

To directly assess the potential influence of FGF15/19 to an obesogenic diet, $\mathrm{Fg} f 15^{+/+}$and $\mathrm{Fgf15^{-/ }}$ mice were fed a 
control or a HFD for 12 weeks. Whereas $F g f 15^{-/-}$mice fed a chow diet gained less weight than $F g f 15^{+/+}$, this outcome was reversed on a HFD, without significant differences in food intake between genotypes (figure 1A). Total body fat and intrahepatic TG content were also markedly higher in Fgf15 mice (figure 1B, C). Hepatic TG levels were also elevated in Fgf $15^{-/-}$mice compared with $F g f 15^{+/+}$, even under a normal diet (figure 1C). While de novo liver fatty acid synthesis from carbohydrate sources is mainly regulated by the transcription factors sterol-regulatory element-binding protein 1c (SREBP1c) and carbohydrate-responsive element-binding protein (ChREBP), ${ }^{1}$ lipid accumulation in diet-induced hepatic steatosis appears to be mainly mediated by peroxisome proliferatoractivated receptor $\gamma$ variant 2 (PPAR $\gamma 2) .{ }^{27}$ Ppar 2 levels are low in the normal liver becoming induced upon HFD feeding. ${ }^{27}$ Accordingly, Ppary 2 expression was upregulated in Fgf $15^{+/+}$mice fed a HFD, and the expression of this gene reached highest levels in FGF15-null mice (figure 1D). Moreover, hepatic Ppary2 expression was already elevated in $\mathrm{Fg} 15^{-/-}$mice fed a control diet (figure 1D). However, we did not observe any significant differences in the hepatic expression of the lipogenic genes Srebp1 and Fas (fatty acid synthase) between $\mathrm{Fgf15^{-/- }}$ and $\mathrm{Fgf15^{+/+ }}$ mice under any feeding conditions (data not shown).

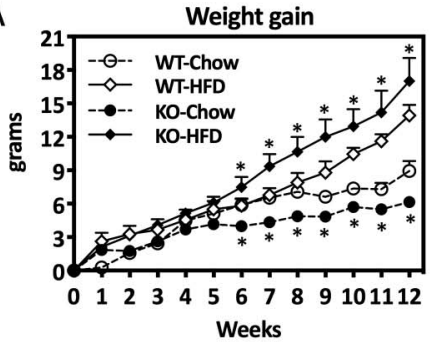

B

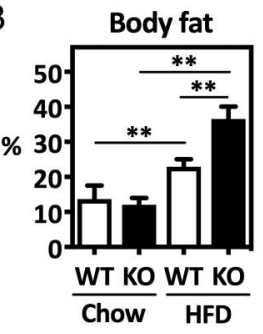

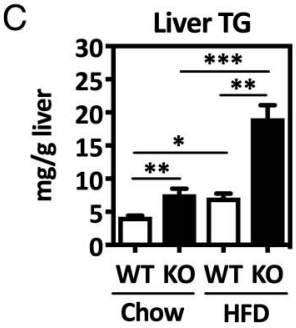

D
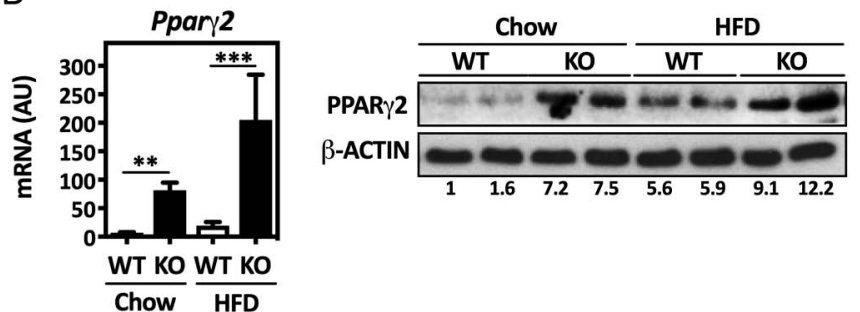

$\mathrm{F}$
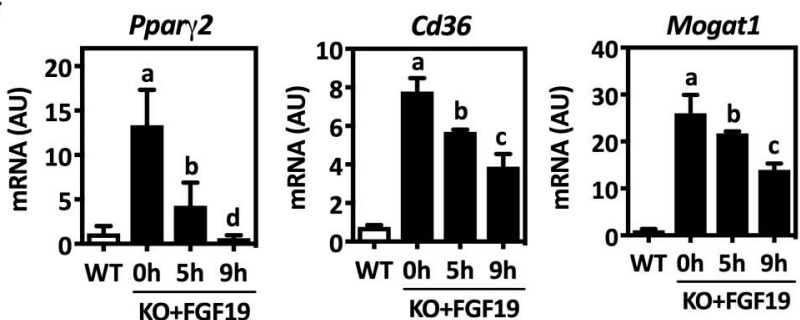

E
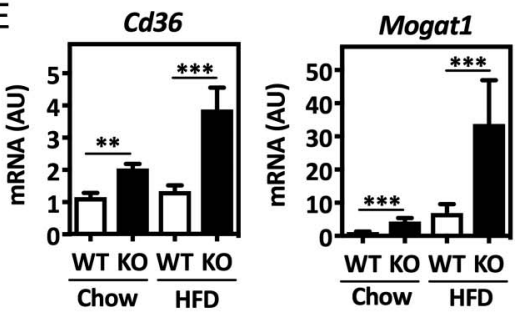

G

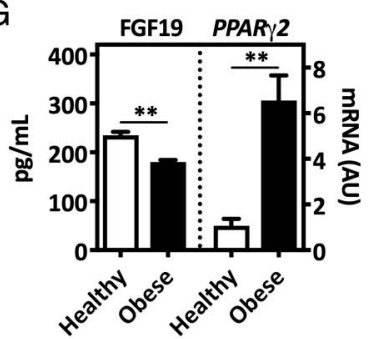

$\mathrm{H}$

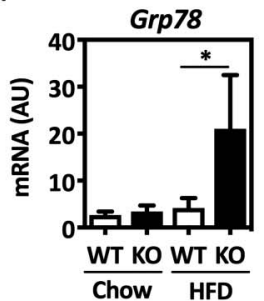

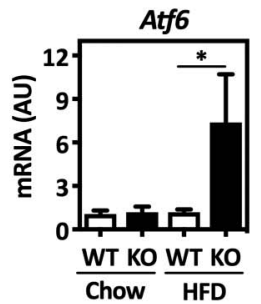

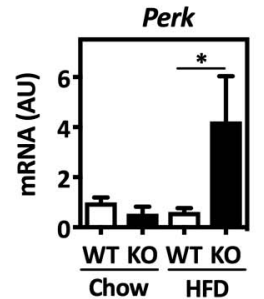

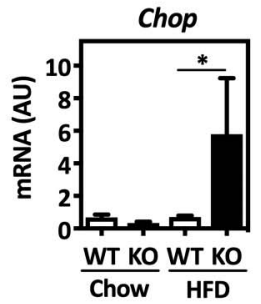

I

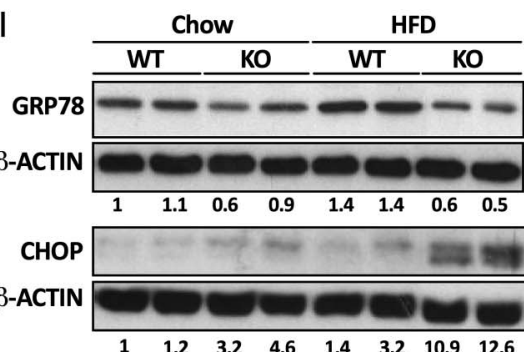

Figure 1 Lack of FGF15/19 leads to increased adiposity and hepatic steatosis accompanied by endoplasmic reticulum (ER) stress in response to a high fat diet (HFD). Weight gain (A), body fat content (B) and liver triglycerides, TG (C) in Fgf15 $5^{+/+}$(wild-type, WT) and Fgf15 $5^{-/-}$mice knockout (KO) after 12 weeks on a chow diet or a HFD. The expression levels of Ppar $\gamma 2$ mRNA (AU, arbitrary units) and PPAR 2 protein (D), and the mRNA levels of the PPAR $\gamma 2$ target genes Cd36 and Mogat1 (E) in the liver of Fgf15 ${ }^{+/+}$(WT) and Fgf15 ${ }^{-1-}$ mice (KO), fed a chow or a HFD for 12 weeks, were determined by qPCR or western blotting. Expression of Ppary2, Cd36 and Mogat1 in the liver of Fgf15 ${ }^{-1-}$ mice (KO) at different time points after recombinant FGF19 administration (1 mg/kg, subcutaneously) (F). Circulating FGF19 levels and hepatic PPAR 2 mRNA expression in samples from healthy $(n=18)$ and obese $(n=69)$ patients as determined by ELISA and QPCR, respectively $(G)$. Expression of genes involved in the ER stress response in the liver of $\mathrm{Fgfl}^{+/+}$(WT) and Fgf15 $5^{-/-}$mice (KO) fed a chow or a HFD for 12 weeks examined at the mRNA (H) and protein levels (I). Representative blots are shown, and quantitation of band intensities (normalised to $\beta$-ACTIN) is indicated. $N=5$ mice per group. ${ }^{*} p<0.05 ;{ }^{* *} p<0.01$; ${ }^{* * *} p<0.001 ;{ }^{a} p<0.05$ vs WT; ${ }^{b} p<0.05$ vs 0 hours; ${ }^{c} p<0.001$ vs 0 hours; ${ }^{d} p<0.0001$ vs 0 hours. 
The biological significance of Ppary2 overexpression was supported by the concomitant upregulation of two key PPAR $\gamma 2$ target genes, Cd36/FAT (fatty acid translocase) and monoacylglycerol O-acyltransferase 1 (Mogat1), involved in liver fat accumulation (figure $1 \mathrm{E}$ and online supplementary figure $\mathrm{S} 1 \mathrm{~A}){ }^{27}$ These findings suggested that FGF15/19 might directly regulate the expression of hepatic Ppary2. To evaluate this possibility, we treated $\mathrm{Fgf15} 5^{-/}$mice with recombinant FGF19 and measured the hepatic expression of Ppary2, Cd36 and Mogat1 at different time points. As shown in figure 1F, FGF19 markedly reduced the expression of these genes. Consistently, Ppar 2 mRNA levels were also reduced in $\mathrm{Fg} f 5^{-/-}$and wild-type mice upon infection with an FGF15-expressing adenovirus (see online supplementary figure S1B). This in vivo effect of FGF19 on Ppary 2 expression is likely to be mediated through a direct interaction with the hepatocyte, as we observed that treatment of cultured human hepatic cells (HepG2) with FGF19 significantly reduced Ppary2 mRNA levels (see online supplementary figure S1C). Some of us recently reported that FGF19 circulating levels are low in obese patients. ${ }^{28}$ Data in figure $1 \mathrm{G}$ confirm this and interestingly show that hepatic PPAR 2 expression was markedly induced in these individuals.

ER stress has been observed both in liver samples from patients with NAFLD and experimental models of fatty liver. $^{1} 2{ }^{29}$ Importantly, chronic ER stress is linked to lipid metabolism dysregulation and fatty liver disease progression. ${ }^{1} 230$ In view of this, we examined the expression of key ER stress response genes in the livers of $\mathrm{Fg} f 15^{+/+}$and $\mathrm{Fgf15^{-/- }}$ mice fed control or HFD for 12 weeks. We found that while $F g f 15^{+/+}$ mice still did not show signs of ER stress, FGF15-null mice on HFD displayed higher mRNA levels of Grp78, Atf6, Perk and C/ EBP homologous proteins (CHOP), indicative of an ongoing ER stress response (figure 1H). ${ }^{19} 3031$ Consistent with the previous findings in $d b / d b$ obese mice, ${ }^{31}$ GRP78 protein levels were reduced in the liver of $\mathrm{Fg} 15^{-/-}$mice, particularly upon HFD feeding, while CHOP levels were induced (figure 1I).

FGF15/19 was recently identified as an ER stress response gene in human colonic cells and mouse ileum. ${ }^{32}$ In that study, FGF15/19 expression was triggered with chemical ER stress inducers in a farnesoid $X$ receptor independent manner. ${ }^{32}$ However, the physiological significance of this response remains unknown. We reproduced the activation of ileal Fgf15 expression, along with that of ER stress response genes, in mice gavaged with the chemical ER stress inducer tunicamycin (figure 2A). In view of this, we speculated that a HFD, particularly a diet rich in saturated FAs which are strong ER stress inducers, ${ }^{2}$ could trigger ileal FGF15/19 expression. We found that mice gavaged with a bolus of palm oil enriched with PA, or with a standard nutrient-rich liquid diet (Ensure), showed the expected peak of ileal Fgf15 expression previously observed

A Ileum
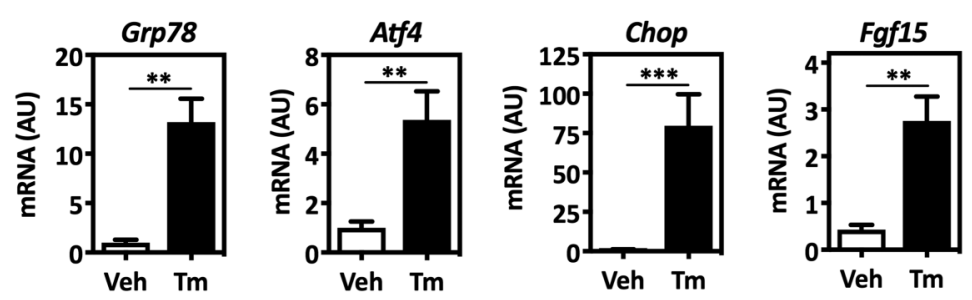

Veh: Vehicle

Tm: Tunicamycin

B

Ileum
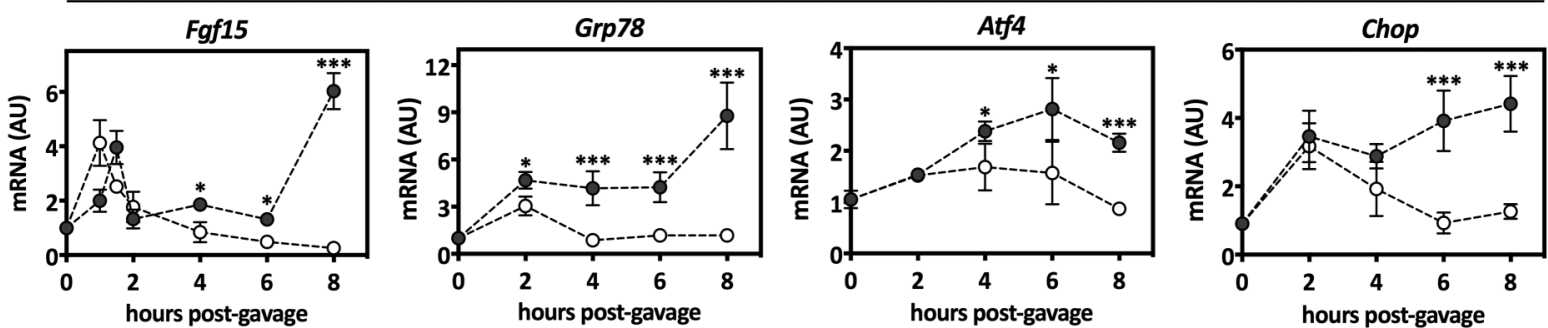

C Liver
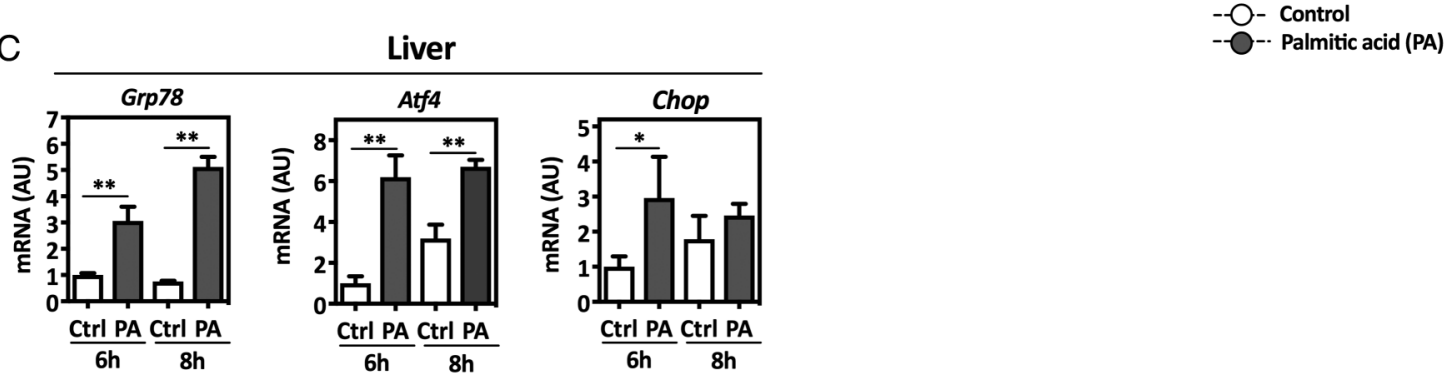

Figure 2 FGF15 expression is induced by endoplasmic reticulum (ER) stress in mouse ileum. Wild-type mice received tunicamycin (Tm) (100 $\mu \mathrm{g} /$ mouse) in a $150 \mathrm{mM}$ sucrose solution, or the same volume of this vehicle (Veh) by gavage, and were sacrificed 6 hours later. The expression of the indicated genes involved in ER stress, and that of Fgf15, was measured by QPCR in ileal tissues (A). Fasted wild-type mice were gavaged with a bolus of control liquid diet or with the same volume of a palmitic acid (PA)-enriched palm oil suspension. At the indicated time points, the expression of ER stress-related genes was determined by $q P C R$ in the ileum (B) or liver (C) of these mice. $N=5$ mice per group. ${ }^{*} p<0.05$; ${ }^{* *} p<0.01$; ${ }^{* * *} p<0.001$. AU, arbitrary units 
between 60 and 90 min post gavage (figure 2B). ${ }^{33}$ However, in PA-gavaged mice, ileal Fgf15 expression remained higher than in control mice at later time points, with a second peak between 6 and 8 hours, coinciding with the sustained activation of ER stress response genes (figure 2B). Concomitantly, increased hepatic expression of ER stress marker genes was observed in PA-gavaged mice compared with the mice fed the standard diet (figure 2C).

FGF19 protects liver cells from lipoapoptosis

Excessive exposure of hepatocytes and cholangiocytes to saturated FAs such as PA triggers ER and oxidative stress, ultimately leading to lipoapoptosis. ${ }^{2}{ }^{6}$ Accordingly, we found that
PA-treated human cholangiocytes (H69 cells) and parenchymal cells (HepG2 cells) underwent apoptosis (figure 3A). This response, together with the activation of caspase 3, was reduced by FGF19 treatment (see figure 3A and online supplementary figure S2A). The expression of the FA transporter CD36 and the FA acyl-CoA activator fatty acid transport protein $4 \mathrm{FATP}^{34}$ was induced upon PA treatment and was also downregulated by FGF19 (figure 3B). Consistent with the cytoprotective effect of FGF19, we observed that the expression of ER stress response genes triggered by PA was attenuated by FGF19 (see figure 3C and online supplementary figure S2B), while the levels of GRP78 protein were preserved (figure 3C). Interestingly, FGF19 expression was induced by PA treatment in $\mathrm{H} 69$ but not in
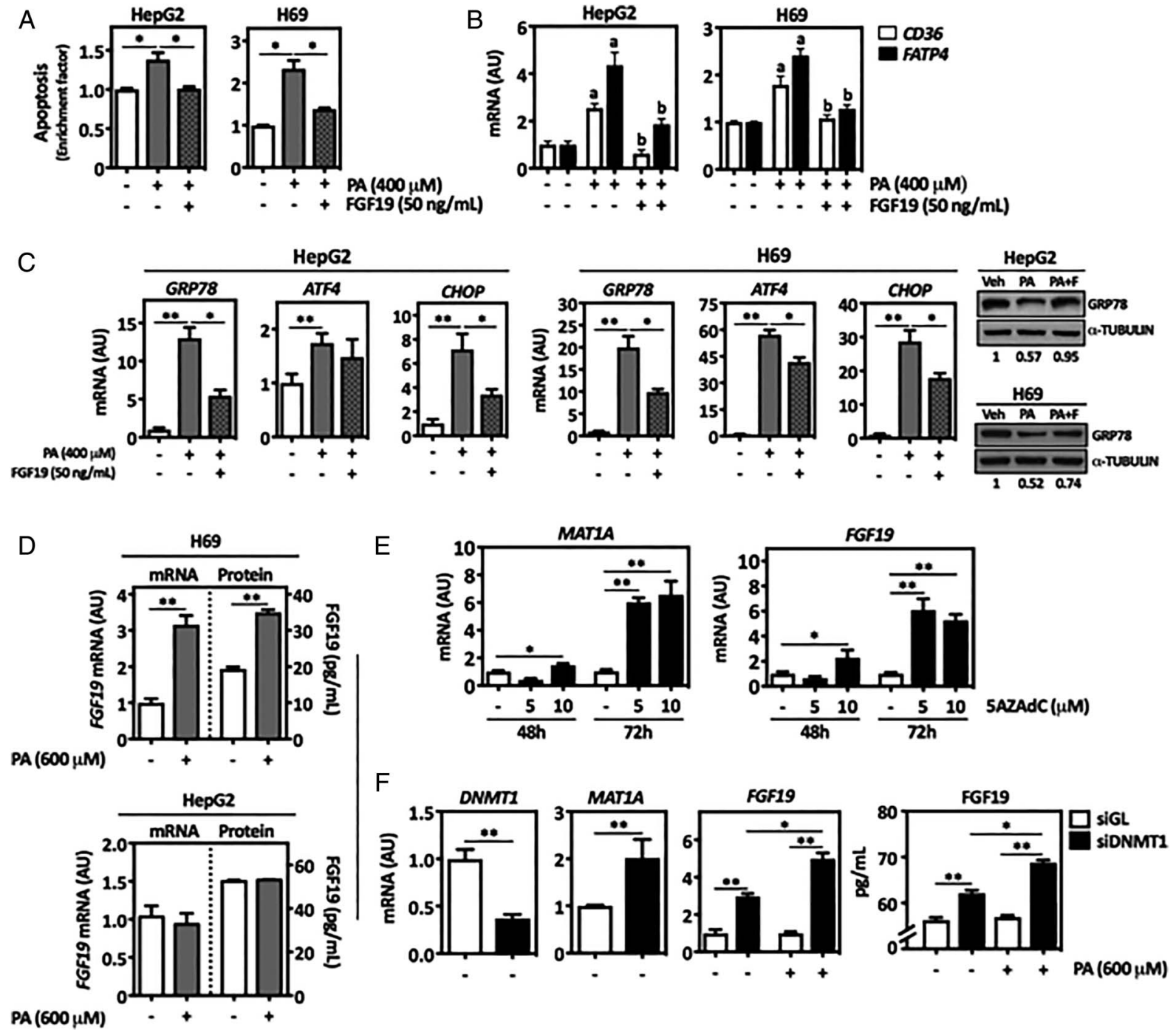

Figure 3 FGF19 protects from lipoapotosis, and its expression is induced by palmitic acid (PA) in liver cells. Human liver parenchymal cell line HepG2 and cholangiocyte cell line $\mathrm{H} 69$ were treated as indicated with PA $(400 \mu \mathrm{M})$ and FGF19 $(50 \mathrm{ng} / \mathrm{mL})$ for 24 hours, and then apoptosis was measured (A). Analysis by qPCR of CD36 and FATP4 mRNA levels in HepG2 and H69 cells treated with PA and FGF19 for 24 hours as indicated above (B). Analysis by qPCR of endoplasmic reticulum (ER) stress-related genes mRNA levels in HepG2 and H69 cells treated with PA and FGF19 for 24 hours as indicated. Right panel show representative western blot analyses of GRP78 protein levels in HepG2 and H69 cells treated as indicated above. Representative blots are shown, and quantitation of band intensities (normalised to $\alpha$-TUBULIN) is indicated (C). H69 and HepG2 cells were treated with PA $(600 \mu \mathrm{M})$ or its vehicle (Veh, $1 \%$ bovine serum albumin (BSA) with $<1 \%$ isopropanol) for 24 hours. Cellular FGF19 mRNA levels were measured by qPCR, and FGF19 protein levels were determined in conditioned media by ELISA (D). HepG2 cells were treated with the DNA demethylating agent 5-aza-deoxycytidine (5AZAdC) as indicated, and the mRNA levels of methionine-adenosyltransferase 1A (MAT1A) and FGF19 were measured by qPCR (E). HepG2 cells were transfected with DNA-methyltransferase 1 (DNMT1)-specific siRNAs, or with control siRNAs (siGL) for 48 hours, and where indicated cells were also treated with PA $(600 \mu \mathrm{M})$ for another 24 hours. The expression of DNMT1, MAT1A and FGF19 mRNAs was measured by $q P C R$, and the levels of FGF19 protein were determined in conditioned media by ELISA (F). ${ }^{*} p<0.05 ;{ }^{* *} p<0.01 ;{ }^{a} p<0.05$ vs Veh; ${ }^{b} p<0.05$ vs $P A$ alone. $A U$, arbitrary units 
HepG2 cells (figure 3D). FGF19 gene expression and inducibility are tissue-specific, and in the enterobiliary tract, it is found in the epithelial cells of the common bile duct, gall bladder and small intestine, but not in the normal liver parenchyma. ${ }^{35} 36$ Epigenetic mechanisms are to a great extent involved in cell type-specific gene expression. Accordingly, treatment of HepG2 cells with the demethylating agent 5-aza-deoxycytidine (5AZAdC) induced FGF19 mRNA levels (figure 3E). The expression of methionine-adenosyltransferase 1A (MAT1A), which is hypermethylated and downregulated in hepatocellular carcinoma (HCC), is shown as control of 5AZAdC treatments. ${ }^{37}$ Similar findings were obtained upon knockdown of DNA-methyltransferase 1 (DNMT1) (figure 3F). Interestingly, in the face of DNMT1 knockdown, PA treatment elicited FGF19 gene expression in HepG2 cells (figure 3F).

\section{Development of the chimaeric FGF19 variant Fibapo and evaluation of its biological activity}

An initial biological characterisation of FA, a fusion protein encompassing FGF19 coupled to human ApoA-I in its C-terminus (see online supplementary figure S3A), was performed by the hydrodynamic injection to mice of plasmids encoding FA or FGF19. Serum half-life of FGF19 was markedly extended upon ApoA-I conjugation, and as expected FA was incorporated into the high density lipoproteins (HDL) fraction of serum lipoproteins (see online supplementary figure S3B, C). To demonstrate the biological activity of FA, we first generated hepatotropic adeno-associated viral vectors (AAV) harbouring FGF19, FA or ApoA-I cDNAs regulated by a hepatocyte-specific promoter (AAV-FGF19, AAV-FA and AAV-ApoA-I). ${ }^{38}$ As shown in figure 4A, serum levels of FA were significantly higher than those of FGF19 in Fgf15 ${ }^{-/}$mice. We examined the tissue distribution of FGF19 and FA and found high levels of both proteins in the liver, the main site of AAV infection, while FA was also detected in white and brown adipose tissue, muscle and brain (figure 4B). FA reproduced the biological effects of FGF19 downregulating hepatic Ppary 2 and Cyp7a1 gene expression, and reducing intrahepatic TG content and serum BA levels in $\mathrm{Fgfl}^{-/-}$mice. Infection with AAV-ApoA-I had no major effects, with the exception of Ppary2 expression (figure 4C, D). Similar effects were observed in obese $d b / d b$ mice, in which infection with AAV-FGF19 or AAV-FA also reduced Ppary2, Mogat1, Cd36 and Fatp4 mRNA levels and intrahepatic TG content, as well as hepatic Cyp7a1 expression and BA levels (see figure 4E, F and online supplementary figure S4). Noteworthy, AAV-ApoA-I also reduced the expression of the genes involved in lipid metabolism tested here, and partially decreased hepatic TG levels (figure 4E). Interestingly, the ER stress response that develops in the liver of $d b / d b$ mice, demonstrated by the low levels of GRP78 protein as described, ${ }^{31}$ was also counteracted by AAV-FGF19 and AAV-FA (figure 4G). Next, we subjected these mice to a model of acute liver failure due to extensive parenchymal resection $(85 \% \mathrm{PH}) .{ }^{21}$ Mice infected with AAV-FA showed significantly enhanced survival (figure $4 \mathrm{H}$ ). We found that the circulating levels of FA measured 6 and 12 hours after $85 \% \mathrm{PH}$ were markedly higher than those of FGF19 (see online supplementary figure S5).

\section{Recombinant Fibapo improves the regeneration of steatotic livers}

Next, we examined the pharmacological properties and potential application of recombinant FA in mouse models of fatty liver regeneration. First, we verified that after equimolar administration, the levels of FA in circulation were more stable in time than those of FGF19, both after intravenous or subcutaneous injections (figure 5A, B). Indeed, serum half-life of FA was 8.5 hours, significantly higher than that of FGF19 (1.8 hours) (see online supplementary figure S6A) ${ }^{24}$ We also analysed the tissue distribution of FA and FGF19 upon intravenous administration. Twenty-four hours after injection, FA was detected in FGF19 target tissues, including liver, brain and adipose tissues, ${ }^{35}$ while FGF19 was only detectable in brown adipose tissue (figure 5C). Similar data were obtained after subcutaneous administration of both proteins (not shown). To examine the biological activity of FA in parallel to FGF19, and ApoA-I as control, we administered a single subcutaneous injection of equimolar amounts of these proteins to $\mathrm{Fgf15^{-/- }}$ mice. FA potently reduced Cyp7a1 expression and circulating BA levels, as well as Ppary2 expression (figure 5D). We also tested FGF19 and FA in $d b / d b$ mice that received three equimolar subcutaneous injections of FA, FGF19 or ApoA-I on alternate days. Forty-eight hours after the last administration, we measured the hepatic expression of Cyp7a1, Ppary2 and Fatp4 along with the intrahepatic levels of BAs and TGs. For all these parameters, FA displayed significantly better activity than FGF19 (figure 5E, F). Interestingly, FA also improved ER stress in $d b / d b$ mice livers, as indicated by GRP78 protein levels (figure 5G). To evaluate whether the beneficial effects of FA on the liver of obese and steatotic mice could improve their outcome after hepatic resection, we performed a $66 \% \mathrm{PH}$ in $d b / d b$ mice that had been treated with FGF19, FA or ApoA-I, as described above. The $d b /$ $d b$ mice show high early mortality and impaired regeneration after $\mathrm{PH} .{ }^{18}$ We reproduced this response and found that all $d b /$ $d b$ mice treated with FA survived the intervention, while a marginal effect was observed in animals that received FGF19 (figure 5H). Survival was monitored in all groups for up to 7 days post $\mathrm{PH}$ without finding any additional changes (not shown). Staining of liver tissues harvested 44 hours after $\mathrm{PH}$ with Ki-67 revealed a marked impairment in cell cycle progression in control $d b / d b$ mice compared with lean (heterozygous) animals, and this was partially recovered by FGF19 and FA treatment, but not by Apo-AI (figure 5I). Nevertheless, besides the effect of FA on mice survival, its overall proregenerative activity appeared superior to the other treatments, as indicated by liver index values 44 hours after $\mathrm{PH}$ that were similar to those found in lean heterozygous mice (figure 5J). FA administration also resulted in reduced intrahepatic BA concentrations and improved liver biosynthetic activity, as indicated by increased levels of serum albumin (see online supplementary figure S6B). Mechanistically, the proregenerative and trophic effects of FA may stem in part from the ability of FGF19 to stimulate protein synthesis in hepatic cells. ${ }^{7}$ Accordingly, we found increased levels of phosphorylated ribosomal protein S6 in the livers of FA-treated mice 44 hours after $\mathrm{PH}$ (see online supplementary figure S6C). Consistent with this observation, both FGF19 and FA, but not ApoA-I, induced the phosphorylation of $\mathrm{p} 70 \mathrm{~S} 6 \mathrm{kinase}(\mathrm{p} 70 \mathrm{~S} 6 \mathrm{~K})$ in human parenchymal liver cells (Hep3B cells), and this response was abrogated by the FGFR4-specific inhibitor BLU9931 (see online supplementary figure S6D).

\section{DISCUSSION}

In addition to its inhibitory effect on hepatic BA synthesis, FGF15/19 has been implicated in the regulation of energy homeostasis. $^{8} 939$ It was early observed that FGF19 transgenic mice showed reduced fat content on a normal diet and were protected from diet-induced obesity. ${ }^{10}$ This phenotype was explained by enhanced energy expenditure due to increased 
A
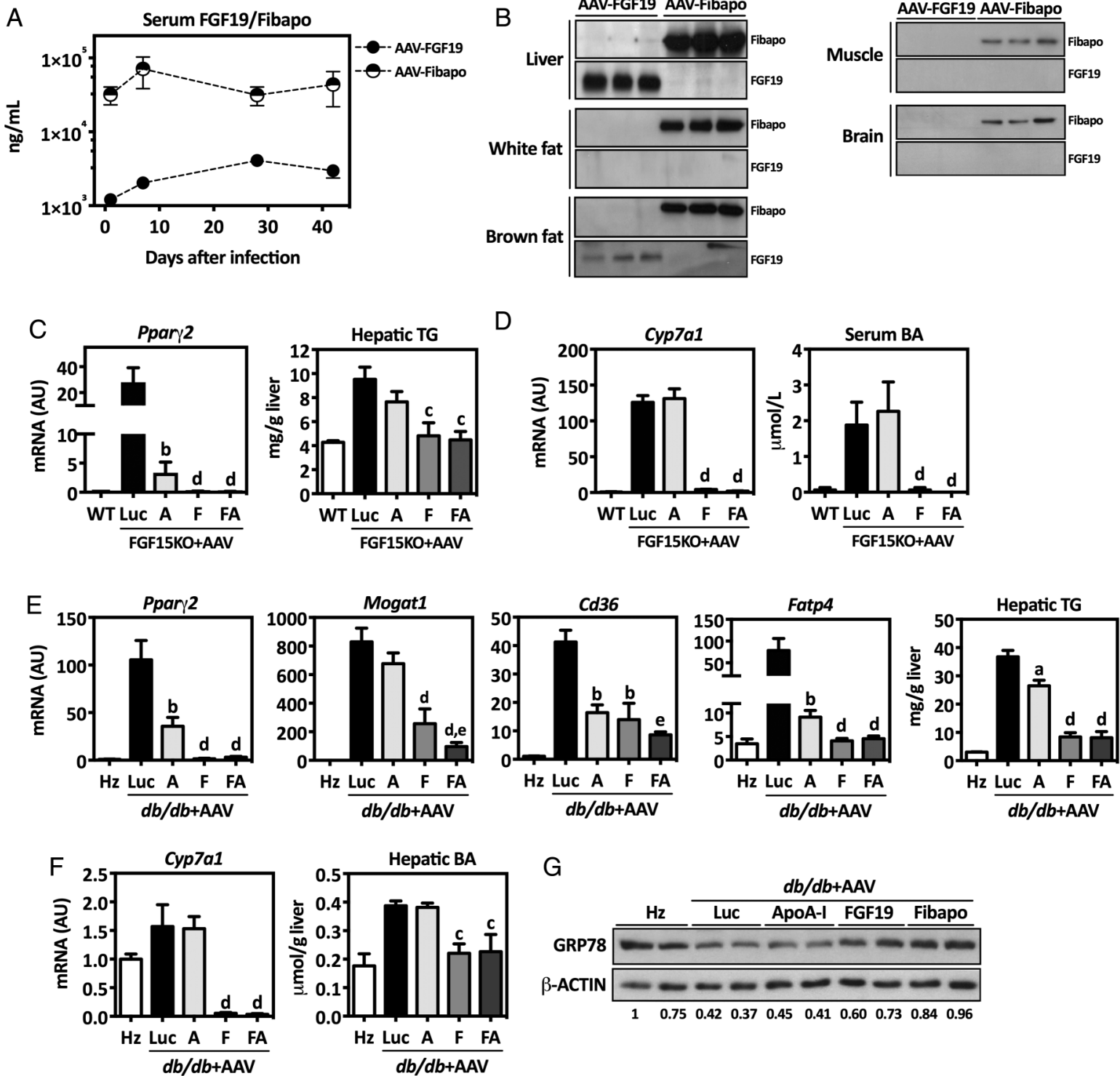

G
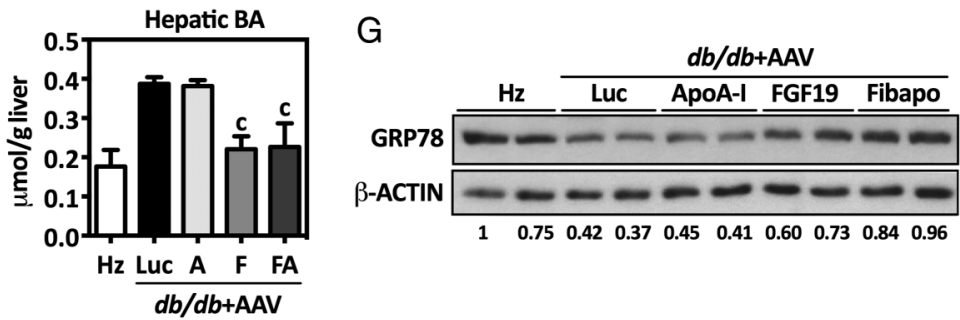
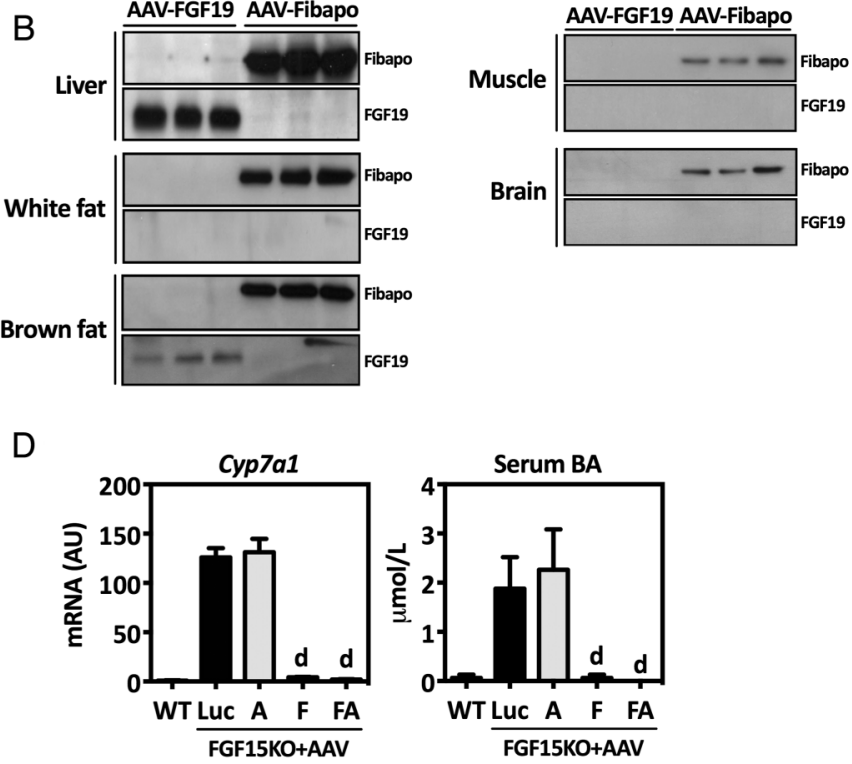

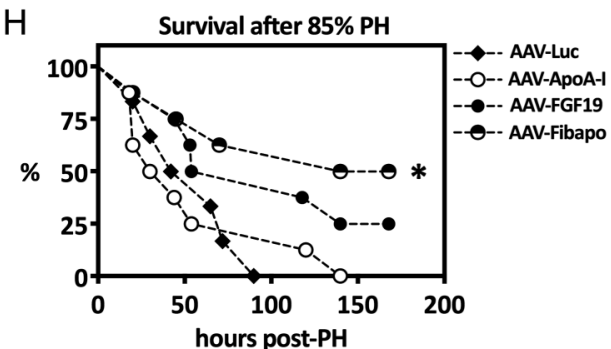

Figure 4 Development of the FGF19-based chimaeric protein Fibapo (FA) and evaluation of its biological activity. Fgf15 ${ }^{-1-}$ mice were infected with adeno-associated viral vectors (AAV) expression vectors encoding FGF19 and FA cDNAs. FGF19 and FA levels were determined in serum by ELISA at the indicated time points (A) or were detected by western blotting in the indicated tissues 28 days after infection (B). Fgf15 ${ }^{-/-}$mice were infected with AAV vectors encoding the control cDNA luciferase (Luc), ApoA-I (A), FGF19 (F) or FA. The hepatic levels of Ppar 2 mRNA and triglycerides (TG) were determined 28 days after infection (C). Expression of Cyp7a1 mRNA in the liver and serum bile acid (BA) levels were also measured in these mice (D). $d b / d b$ mice were infected with the AAV vectors described above. Ten days after infection, the expression of the indicated genes along with TG levels were measured in the liver (E). The expression of Cyp7a1 and hepatic BA contents were also examined in these liver tissues (F). The expression of GRP78 was determined by western blotting in liver samples from control mice (db/+, heterozygous mice, $\mathrm{Hz}$ ) and $d b / d b$ mice infected with the indicated AAVs. Representative blots are shown, and quantitation of band intensities (normalised to $\beta$-ACTIN) is indicated (G). Survival of $d b / d b$ mice that were infected with the indicated AAVs and 10 days later were subjected to $85 \%$ partial hepatectomy (PH) (at least 10 mice were used per condition) (H). ${ }^{a} p<0.05$ vs AAV-Luc; ${ }^{b} p<0.01$ vs AAV-Luc; ${ }^{c} p<0.05$ vs AAV-ApoA-I; ${ }^{d} p<0.01$ vs AAV-ApoA-l; ${ }^{e} p<0.05$ vs AAV-FGF19 and AAV-ApoA-I; * $\mathrm{p}<0.05$ vs AAV-Luc and AAV-ApoA-I. AU, arbitrary units. 

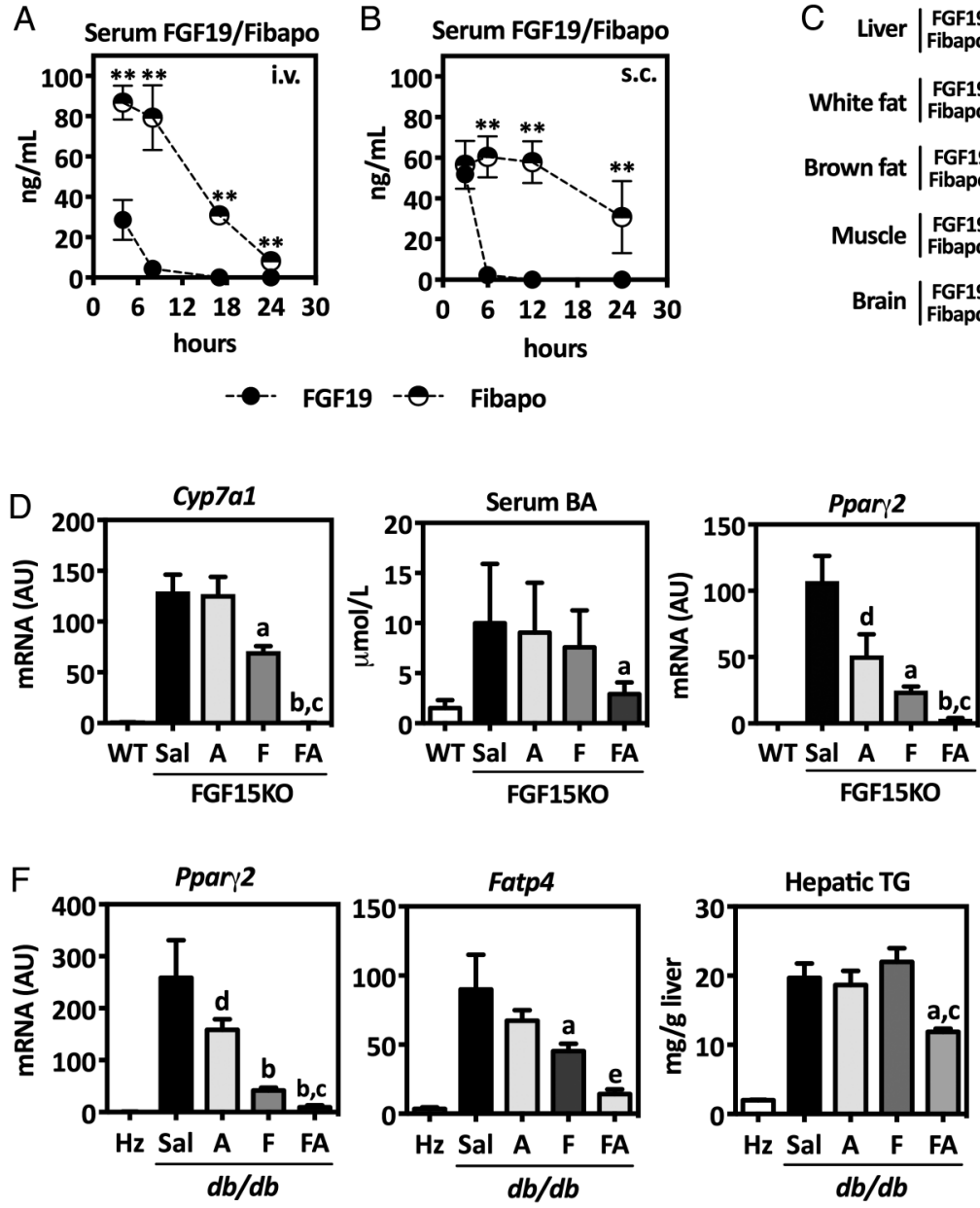

G
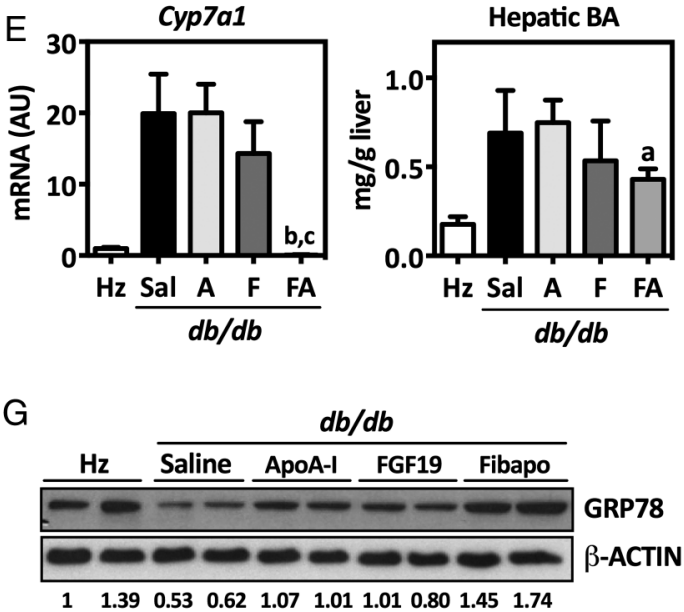

$\mathrm{H}$

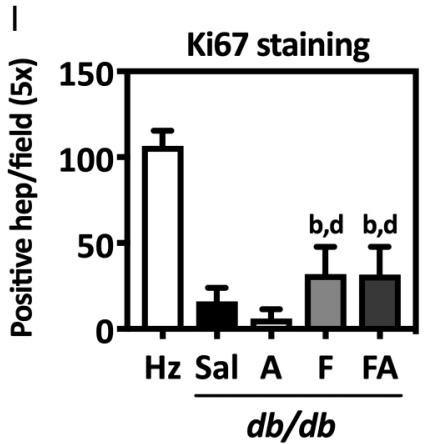

Figure 5 Fibapo (FA) shows improved pharmacological properties than FGF19 and is effective in preventing postresection liver failure in mice with fatty livers. Serum levels of FGF19 and FA determined by ELISA at different time points after injection of equimolar amounts of both proteins (45 nmol/kg body weight) intravenously (A) or subcutaneously (B) in Fgf15 ${ }^{-/-}$mice. Tissue distribution of recombinant FGF19 and FA in different mouse tissues 24 hours after intravenous administration of equimolar amounts of both proteins as determined by ELISA (C). Effect of a single administration to $\mathrm{Fgf15}^{-/-}$mice of equimolar amounts of ApoA-I, FGF19 or FA (45 nmol/kg body weight) on liver Cyp7a1 mRNA expression, serum bile acids (BA) levels and Ppar 2 mRNA levels examined 24 hours after protein injections. Values in Fgf15 ${ }^{+/+}$(wild-type, WT) mice are shown as controls (D). Effect of the administrations to $d b / d b$ mice of equimolar amounts of ApoA-I, FGF19 or FA (45 nmol/kg body weight) on liver Cyp7a1 mRNA expression and BA levels examined 48 hours after protein injections. Values in $d b /+$ (heterozygous, $\mathrm{Hz}$ ) mice are shown as controls (E). Determination of Ppary2 and Fatp4 mRNA and triglycerides (TG) levels in the livers of $d b / d b$ mice treated as indicated above (F). Expression levels of GRP78 protein determined by western blotting in the livers of $d b / d b$ mice treated with ApoA-I, FGF19 and FA as described above. Representative blots are shown, and quantitation of band intensities (normalised to $\beta$-ACTIN) is indicated (G). Heterozygous $d b /+$ and $d b / d b$ mice ( $N=5$ mice per group) treated as indicated above underwent partial hepatectomy (PH) (66\%) 48 hours after the last administration of the recombinant proteins (or saline, Sal) and survival was monitored up to 44 hours post PH (H). Quantification of Ki67 staining in liver tissue sections taken from mice 44 hours post PH (I). Liver to body weight ratio after 44 hours post PH (J). ${ }^{*} p<0.05 ;{ }^{*} p<0.01 ;{ }^{a} p<0.05$ vs ApoA-I; ${ }^{b} p<0.01$ vs ApoA-I; ${ }^{C} p<0.01$ vs FGF19; ${ }^{d} p<0.05$ vs saline; ${ }^{e} p<0.05$ vs FGF19; ${ }^{f} p<0.05$ vs all other $d b / d b$ groups. $A U$, arbitrary units. 
brown adipose tissue mass, as well as by reduced lipogenesis and enhanced hepatic lipid oxidation. ${ }^{10}$ Glucose homeostasis and insulin sensitivity were also improved in FGF19 transgenic mice or upon recombinant FGF19 administration to obese mice. ${ }^{10} 11$ These responses apparently involve the action of FGF19 on different tissues, including adipose tissue, liver and also the central nervous system. ${ }^{9}$ The important effects of FGF15/19 on glucose metabolism, including stimulation of glycogen synthesis and inhibition of gluconeogenesis, were recently demonstrated through more physiological approaches using FGF15-null mice. ${ }^{73}$ Now we provide novel evidence on the essential role of endogenous FGF15 in fat metabolism, particularly in the adaptation to high dietary fat contents. Interestingly, under a chow diet, we found that $\mathrm{Fgf15}^{-/-}$animals gained less weight than wild types. Although we do not have a specific explanation for this difference, we may especulate that lack of FGF15/19, a relevant hormone governing nutrient metabolism, may somehow impair the physiological weight gain of young adult mice. Conversely, $\mathrm{Fgf} 15^{-/-}$mice showed increased weight gain, exacerbated systemic adiposity and hepatosteatosis when fed a HFD. Previous in vitro experiments demonstrated that FGF19 counteracted the upregulation of lipogenic genes such as Srebp1 and Fas elicited by insulin in cultured hepatocytes. ${ }^{40}$ As mentioned before, we did not observe significant differences in liver Srebp1 and Fas expression between $\mathrm{Fgf15^{-/- }}$ and $\mathrm{Fgf15} 5^{+/+}$mice. However, we found that lack of FGF15 resulted in a marked upregulation of hepatic Ppary2 expression, a response that was exacerbated upon HFD feeding. Ppary 2 is a transcription factor expressed at very low levels in normal human and mouse hepatocytes, induced in models of obesity and steatosis, and involved in the upregulation of adipogenic genes such as $C d 36$ and Mogat $1 .^{27}$ Importantly, hepatic overexpression of Ppary 2 and Mogat1 can drive liver steatosis even under a normal diet. ${ }^{27}{ }^{41}$ While the influence of FGF15 on metabolic regulation in extrahepatic tissues (eg, adipose tissue) is likely relevant for systemic lipid metabolism, ${ }^{39} 42$ we believe that the repressive effect of FGF15/19 on liver Ppary 2 expression may be relevant for the metabolic adaptation to fat intake. In support of this, we found that exogenous FGF15/19 administration inhibited hepatic Ppary 2 expression and function (ie, Ppary 2 target genes expression) and that the expression of PPAR 2 was markedly induced in the liver of obese patients, who also show reduced FGF19 circulating levels. ${ }^{28}$ Ppar 2 promoter regulation in the liver is not completely understood, but the involvement of transcriptional regulators from the Activator Protein 1 family, as well as Small Heterodimer Partner and Hepatocyte Nuclear Factor $4 \alpha$, all of them downstream targets of FGF15/19 signalling, ${ }^{8}$ has been demonstrated. ${ }^{43}{ }^{44}$ According to our findings, FGF15/19 would be the first identified physiological signal driven by nutritional cues that can modulate hepatic Ppary2 expression.

At variance with wild-type animals, $\mathrm{Fgf15^{-/- }}$ mice developed signs of hepatic ER stress in our HFD model. Initially, the ER stress response may be an adaptive reaction to lipid overload; however, protracted ER stress has been associated with NAFLD progression. The ER is highly sensitive to excess lipids, and saturated FAs in particular promote ER stress ultimately leading to apoptosis of liver cells. ${ }^{1}{ }^{2}$ Intriguingly, Fgf15 expression can be triggered by non-physiological activators of the ER stress response in mouse ileum. ${ }^{32}$ We found that acute feeding of a PA-enriched lipid mixture induced a potent ileal ER stress response and sustained Fgf15 expression, which was followed by signs of hepatic ER stress. It was recently demonstrated that the hepatic expression of $\beta$-Klotho, which together with FGFR4 forms the FGF15/19 receptor complex in hepatocytes, ${ }^{35}$ is induced in response to ER stress. ${ }^{45}$ Collectively, these observations suggest that upon increased fat intake, the enhanced release of FGF15/19 from the gut into the portal circulation, together with the induction of $\beta$-Klotho expression in hepatocytes, could facilitate the adaptation of hepatic metabolism to dietary lipid excess in a coordinated manner. Additionally, FGF15/19 has direct cytoprotective effects, as demonstrated in HCC cells. ${ }^{46}$ We found that FGF19 protected HepG2 and H69 cells from lipoapoptosis induced by saturated FAs. The mechanisms involved in this antiapoptotic activity can be multifarious, since FGF15/19 besides a hormone is also a growth factor that activates intracellular survival pathways. ${ }^{8}$ Nevertheless, we found that the upregulation of the FAT CD36 upon PA challenge was inhibited by FGF19 in both cell types. Overexpression of CD36 is associated with increased steatosis and apoptosis in the liver of patients with NAFLD. ${ }^{47}$ Perhaps, a more compelling mechanistic finding was the repression of FATP4 by FGF19 treatment. FATP4 expression is induced in the fatty liver, localising to the ER and mitochondria. This acyl-CoA synthetase catalyses the incorporation of saturated FAs into ER stress-inducing phospholipids and their conversion into other lipotoxic metabolites, mediating to a great extent PA-induced hepatocyte apoptosis. ${ }^{34}$ Consistently, in addition to enhanced cell survival, we also found reduced expression of ER stress marker genes in PA-treated cells that received FGF19. Interestingly, we observed that PA induced the expression of FGF19 in H69 cholangiocytes, but not in the hepatic parenchymal cell line HepG2. This response could represent a protective autocrine/paracrine loop to avoid cholangiocyte lipoapoptosis, ${ }^{6}$ similar to the upregulation of FGF19 in biliary epithelial cells in response to excessive BA concentrations. ${ }^{36} 48 \quad 49$ FGF19 gene expression seems to be differentially controlled in human biliary cells and hepatocytes. FGF19 behaves as a readily inducible gene in cholangiocytes compared with differentiated parenchymal cells, in which it is normally a silenced gene. ${ }^{36} 50$ Epigenetic mechanisms such as DNA methylation greatly influence cell type-specific gene expression. Accordingly, we found that inhibition of DNA methylation promoted FGF19 expression in HepG2 cells and made this gene responsive to PA stimulation. Considering that HCCs are characterised by global DNA hypomethylation, our findings could have broader implications, as they may contribute to explain the overexpression of FGF19 in a significant proportion of HCCs in which the FGF19 gene is not amplified. ${ }^{46}$ Moreover, these observations would be consistent with the recent finding of increased hepatocellular FGF19 expression in patients with chronic liver disease and steatohepatitis. ${ }^{51}$

The complement of biological activities encompassed by FGF19 including the downregulation of hepatic BA synthesis and lipid accumulation, its mitogenic and cytoprotective effects, and its capacity to stimulate protein synthesis could make FGF19 an ideal therapeutic molecule in the context of liver regeneration. Certainly, cholestasis and steatosis are factors that negatively influence organ recovery and regeneration after $\mathrm{PH}$ or transplantation in humans. ${ }^{13-16}$ Moreover, increased ER stress and PA overload are observed in steatotic livers after experimental $\mathrm{PH}$, and modulation of ER stress improves regeneration in fatty livers. ${ }^{19} 20$ Therefore, the protective effects of FGF15/19 described here on steatosis-associated ER stress, as well as on PA-induced cell death, may be of particular relevance in this context. However, as we and others have observed, FGF19 has a very short half-life in circulation. ${ }^{24}$ To overcome this limitation and to enhance FGF19 hepatotropism, we 
developed FA. In addition to fulfilling these expected kinetic and biodistribution properties, FA retained FGF19 inhibitory activities on BA synthesis and also reduced hepatic Ppary2 expression and TG contents. Its administration to obese mice improved survival after extensive (lethal) $\mathrm{PH}$, and enhanced regeneration and promoted survival upon standard (66\%) $\mathrm{PH}$. These effects were observed when FA was expressed from AAV vectors, suggesting its potential administration using gene therapy strategies, and also when it was delivered as a recombinant protein. Interestingly, FA showed better therapeutic activity than FGF19 in the different models of liver steatosis and regeneration tested in our study. While part of this improved performance may be attributed to the enhanced pharmacokinetic properties conferred by ApoA-I, this moiety could also contribute to the therapeutic effects of FA. In fact, ApoA-I administration has been shown to reduce hepatosteatosis and to improve liver histology in obese mice, which is consistent with our observations in $d b / d b$ mice. ${ }^{52}$ Moreover, the interaction of ApoA-I with its receptors SR-BI and ABCA1 expressed in hepatocytes triggers a variety of prosurvival signalling mechanisms. ${ }^{52}$ On the other hand, interaction of HDL-residing FA with SR-BI receptors through its ApoA-I moiety might also result in its internalisation in the cells. This might compromise the binding and activation of the FGFR4/ $\beta$-Klotho complex, leading to a reduced biological activity. Nevertheless, while this situation may indeed occur, our in vivo observations still demonstrate a better pharmacological performance of FA compared with FGF19.

In summary, we have provided novel evidences on the important physiological role played by FGF15/19 in liver fat metabolism, and in protection from lipid-mediated cellular stress and injury. Our observations demonstrated that the biological activities present in FGF15/19 can be harnessed in a new molecule termed Fibapo with improved kinetic and pharmacological properties. As recently proposed for growth hormone, ${ }^{53}$ the perioperative administration of $\mathrm{FA}$, alone or in combination with other strategies such as weight loss, could improve the outcome of patients with steatotic organs that need resection.

Acknowledgements The authors thank Dr Laura Guembe, from CIMA Morphology core facility, for histological and immunohistological analyses.

Contributors GA-S, IU, MUL, RU, ME, MB-V, MJ, HCC and CR-O performed in vitro and in vivo experiments and collected data. VC, AR, JMG-E and AG-N analysed human samples and collected data. GA-S, IU, FJC, JP, PB, FV, GF, CB and MAA designed experiments and discussed the data. IU, CB and MAA wrote and submitted the manuscript.

Funding This work was funded by CIBERehd; Grants FIS PI13/00359, PI13/00385 and PI16/01126 from Instituto de Salud Carlos III (ISCIII), co-financed by 'Fondo Europeo de Desarrollo Regional' (FEDER) 'Una manera de hacer Europa'.

'Adipoplast' Network (BFU2015-70454-REDT). 'Ramón y Cajal-I3' contract to MUL. Marie Curie EU contract to MGF-B. ADA-University of Navarra fellowship to MJ; FPI fellowship from Ministerio de Economía to MB-V; Fundación Eugenio Rodríguez Pascual; Fundación M Torres; Fundación Mario Losantos; Fundación Familia Puig-Infante. We also thank Mr. Eduardo Avila Zaragozá for his generous support.

Competing interests Not declared.

Ethics approval The University Clinic of Navarra and the Santa Creu i Sant Pau Hospital Ethics.

Provenance and peer review Not commissioned; externally peer reviewed.

\section{REFERENCES}

1 Hardy T, Oakley F, Anstee QM, et al. Nonalcoholic Fatty Liver Disease: Pathogenesis and Disease Spectrum. Annu Rev Pathol 2016;11:451-96.

2 Mota M, Banini BA, Cazanave SC, et al. Molecular mechanisms of lipotoxicity and glucotoxicity in nonalcoholic fatty liver disease. Metab Clin Exp 2016;65:1049-61.

3 Kumashiro N, Erion DM, Zhang D, et al. Cellular mechanism of insulin resistance in nonalcoholic fatty liver disease. Proc Natl Acad Sci USA 2011;108:16381-5.
4 Bechmann LP, Kocabayoglu P, Sowa JP, et al. Free fatty acids repress small heterodimer partner (SHP) activation and adiponectin counteracts bile acid-induced liver injury in superobese patients with nonalcoholic steatohepatitis. Hepatology 2013; 57:1394-406

5 Aranha MM, Cortez-Pinto $\mathrm{H}$, Costa A, et al. Bile acid levels are increased in the liver of patients with steatohepatitis. Eur J Gastroenterol Hepatol 2008;20:519-25.

6 Natarajan SK, Ingham SA, Mohr AM, et al. Saturated free fatty acids induce cholangiocyte lipoapoptosis. Hepatology 2014;60:1942-56.

7 Kir S, Beddow SA, Samuel VT, et al. FGF19 as a postprandial, insulin-independent activator of hepatic protein and glycogen synthesis. Science 2011;331:1621-4.

8 Jahn D, Rau M, Hermanns HM, et al. Mechanisms of enterohepatic fibroblast growth factor 15/19 signaling in health and disease. Cytokine Growth Factor Rev 2015;26:625-35

9 Potthoff MJ, Kliewer SA, Mangelsdorf DJ. Endocrine fibroblast growth factors 15/19 and 21: from feast to famine. Genes Dev 2012;26:312-24.

10 Tomlinson E, Fu L, John L, et al. Transgenic mice expressing human fibroblast growth factor-19 display increased metabolic rate and decreased adiposity. Endocrinology 2002;143:1741-7.

11 Fu L, John LM, Adams SH, et al. Fibroblast growth factor 19 increases metabolic rate and reverses dietary and leptin-deficient diabetes. Endocrinology 2004; 145:2594-603.

12 Schreuder TCMA, Marsman HA, Lenicek M, et al. The hepatic response to FGF19 is impaired in patients with nonalcoholic fatty liver disease and insulin resistance. Am J Physiol Gastrointest Liver Physiol 2010;298:G440-5.

13 McCormack L, Petrowsky H, Jochum W, et al. Hepatic steatosis is a risk factor for postoperative complications after major hepatectomy: a matched case-control study. Ann Surg 2007;245:923-30.

14 Kele PG, van der Jagt EJ, Gouw ASH, et al. The impact of hepatic steatosis on liver regeneration after partial hepatectomy. Liver Int 2013;33:469-75.

15 Hoppe S, von Loeffelholz C, Lock JF, et al. Nonalcoholic steatohepatits and liver steatosis modify partial hepatectomy recovery. J Invest Surg 2015;28:24-31.

16 Cho JY, Suh KS, Lee HW, et al. Hepatic steatosis is associated with intrahepatic cholestasis and transient hyperbilirubinemia during regeneration after living donor liver transplantation. Transp/ Int 2006;19:807-13.

17 Yang SQ, Lin HZ, Mandal AK, et al. Disrupted signaling and inhibited regeneration in obese mice with fatty livers: implications for nonalcoholic fatty liver disease pathophysiology. Hepatology 2001;34:694-706.

18 Murata $\mathrm{H}$, Yagi T, Iwagaki $\mathrm{H}$, et al. Mechanism of impaired regeneration of fatty live in mouse partial hepatectomy model. J Gastroenterol Hepatol 2007;22:2173-80.

19 Hamano $\mathrm{M}$, Ezaki $\mathrm{H}$, Kiso S, et al. Lipid overloading during liver regeneration causes delayed hepatocyte DNA replication by increasing ER stress in mice with simple hepatic steatosis. J Gastroenterol 2014;49:305-16.

20 Inaba Y, Furutani T, Kimura K, et al. Growth arrest and DNA damage-inducible 34 regulates liver regeneration in hepatic steatosis in mice. Hepatology 2015;61:1343-56.

21 Uriarte I, Fernandez-Barrena MG, Monte MJ, et al. Identification of fibroblast growth factor 15 as a novel mediator of liver regeneration and its application in the prevention of post-resection liver failure in mice. Gut 2013:62:899-910.

22 Kong B, Huang J, Zhu Y, et al. Fibroblast growth factor 15 deficiency impairs liver regeneration in mice. Am J Physiol Gastrointest Liver Physiol 2014;306:G893-902.

23 Padrissa-Altés S, Bachofner M, Bogorad RL, et al. Control of hepatocyte proliferation and survival by Fgf receptors is essential for liver regeneration in mice. Gut 2015:64:1444-53

24 Luo J, Ko B, Elliott M, et al. A nontumorigenic variant of FGF19 treats cholestatic liver diseases. Sci Trans/ Med 2014;6:247ra100.

25 Fioravanti J, González I, Medina-Echeverz J, et al. Anchoring interferon alpha to apolipoprotein $\mathrm{A}$-I reduces hematological toxicity while enhancing immunostimulatory properties. Hepatology 2011;53:1864-73.

26 Uriarte I, Latasa MU, Carotti S, et al. Ileal FGF15 contributes to fibrosis-associated hepatocellular carcinoma development. Int J Cancer 2015;136:2469-75.

27 Lee YJ, Ko EH, Kim JE, et al. Nuclear receptor PPAR -regulated monoacylglycerol 0 -acyltransferase 1 (MGAT1) expression is responsible for the lipid accumulation in diet-induced hepatic steatosis. Proc Natl Acad Sci USA 2012;109:13656-61.

28 Gallego-Escuredo JM, Gómez-Ambrosi J, Catalan V, et al. Opposite alterations in FGF21 and FGF19 levels and disturbed expression of the receptor machinery for endocrine FGFs in obese patients. Int J Obes (Lond) 2015;39:121-9.

29 Leamy AK, Egnatchik RA, Young JD. Molecular mechanisms and the role of saturated fatty acids in the progression of non-alcoholic fatty liver disease. Prog Lipid Res 2013;52:165-74.

30 Kammoun HL, Chabanon H, Hainault I, et al. GRP78 expression inhibits insulin and ER stress-induced SREBP-1C activation and reduces hepatic steatosis in mice. J Clin Invest 2009;119:1201-15.

31 Yamagishi N, Ueda T, Mori A, et al. Decreased expression of endoplasmic reticulum chaperone GRP78 in liver of diabetic mice. Biochem Biophys Res Commun 2012:417:364-70.

32 Shimizu M, Li J, Maruyama R, et al. FGF19 (fibroblast growth factor 19) as a novel target gene for activating transcription factor 4 in response to endoplasmic reticulum stress. Biochem J 2013;450:221-9. 
33 Potthoff MJ, Boney-Montoya J, Choi M, et al. FGF15/19 Regulates Hepatic Glucose Metabolism by Inhibiting the CREB-PGC-1 $\alpha$ Pathway. Cell Metab 2011;13: 729-38.

34 Seeßle J, Liebisch G, Schmitz G, et al. Palmitate activation by fatty acid transport protein 4 as a model system for hepatocellular apoptosis and steatosis. Biochim Biophys Acta 2015;1851:549-65.

35 Fon Tacer K, Bookout AL, Ding X, et al. Research resource: Comprehensive expression Atlas of the fibroblast growth factor system in adult mouse. $\mathrm{Mol}$ Endocrinol 2010;24:2050-64.

36 Zweers SJLB, Booij KAC, Komuta $M$, et al. The human gallbladder secretes fibroblast growth factor 19 into bile: towards defining the role of fibroblast growth factor 19 in the enterobiliary tract. Hepatology 2012;55:575-83.

37 Avila MA, Berasain C, Torres L, et al. Reduced mRNA abundance of the main enzymes involved in methionine metabolism in human liver cirrhosis and hepatocellular carcinoma. J Hepatol 2000;33:907-14.

38 Elizalde M, Urtasun R, Azkona M, et al. Splicing regulator SLU7 is essential for maintaining liver homeostasis. J Clin Invest 2014;124:2909-20.

39 Markan KR, Potthoff MJ. Metabolic fibroblast growth factors (FGFs): mediators of energy homeostasis. Semin Cell Dev Biol 2016;53:85-93.

40 Bhatnagar S, Damron HA, Hillgartner FB. Fibroblast growth factor-19, a novel factor that inhibits hepatic fatty acid synthesis. J Biol Chem 2009;284:10023-33.

$41 \mathrm{Yu}$ JH, Song SJ, Kim A, et al. Suppression of PPAR $\gamma$-mediated monoacylglycerol 0 -acyltransferase 1 expression ameliorates alcoholic hepatic steatosis. Sci Rep 2016:6:29352.

42 Wu X, Ge H, Baribault H, et al. Dual actions of fibroblast growth factor 19 on lipid metabolism. J Lipid Res 2013;54:325-32.

43 Hasenfuss SC, Bakiri L, Thomsen MK, et al. Regulation of steatohepatitis and PPAR $\gamma$ signaling by distinct AP-1 dimers. Cell Metab 2014;19:84-95.
44 Kim SC, Kim CK, Axe D, et al. All-trans-retinoic acid ameliorates hepatic steatosis in mice by a novel transcriptional cascade. Hepatology 2014;59:1750-60.

45 Dong $\mathrm{K}$, Li H, Zhang M, et al. Endoplasmic reticulum stress induces up-regulation of hepatic $\beta$-Klotho expression through ATF4 signaling pathway. Biochem Biophys Res Commun 2015:459:300-5.

46 Hagel M, Miduturu C, Sheets M, et al. First Selective Small Molecule Inhibitor of FGFR4 for the Treatment of Hepatocellular Carcinomas with an Activated FGFR4 Signaling Pathway. Cancer Discov 2015;5:424-37.

47 Bechmann LP, Gieseler RK, Sowa JP, et al. Apoptosis is associated with CD36/fatty acid translocase upregulation in non-alcoholic steatohepatitis. Liver Int 2010;30:850-9.

48 Schaap FG, van der Gaag NA, Gouma DJ, et al. High expression of the bile salt-homeostatic hormone fibroblast growth factor 19 in the liver of patients with extrahepatic cholestasis. Hepatology 2009;49:1228-35.

49 Jung D, York JP, Wang L, et al. FXR-induced secretion of FGF15/19 inhibits CYP27 expression in cholangiocytes through p38 kinase pathway. Pflugers Arch 2014;466:1011-19.

50 Naugler WE, Tarlow BD, Fedorov LM, et al. Fibroblast growth factor signaling controls liver size in mice with humanized livers. Gastroenterology 2015;149:728-40.e15.

51 Li Y, Zhang W, Doughtie A, et al. Up-regulation of fibroblast growth factor 19 and its receptor associates with progression from fatty liver to hepatocellular carcinoma. Oncotarget 2016;7:52329-39.

52 Nofer JR. Signal transduction by HDL: agonists, receptors, and signaling cascades. Handb Exp Pharmacol 2015;224:229-56.

53 Collin de l'Hortet A, Zerrad-Saadi A, Prip-Buus C, et al. GH administration rescues fatty liver regeneration impairment by restoring GH/EGFR pathway deficiency. Endocrinology 2014;155:2545-54. 